\title{
Randomized clinical trial to assess the effect of antibiotic therapy on health and growth of preweaned dairy calves diagnosed with respiratory disease using respiratory scoring and lung ultrasound
}

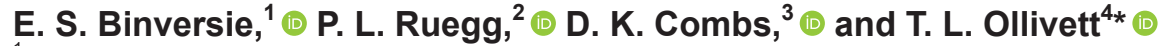 \\ ${ }^{1}$ Saskatoon Colostrum Company Ltd. (SCCL), Mesa, AZ 85206 \\ ${ }^{2}$ Department of Animal Science, Michigan State University, East Lansing 48824 \\ ${ }^{3}$ Department of Animal Science, College of Agricultural and Life Sciences, University of Wisconsin, Madison 53706 \\ ${ }^{4}$ Department of Medical Sciences, School of Veterinary Medicine, University of Wisconsin, Madison 53706
}

\begin{abstract}
The primary objective of this randomized field study was to assess the effect of antibiotic therapy on health and growth of preweaned dairy calves diagnosed with naturally occurring respiratory disease, using respiratory scoring and portable lung ultrasound. A secondary objective was to determine whether treatment response depended on clinical presentation at the time of diagnosis. Holstein calves $(\mathrm{n}=357)$ were enrolled at 3 to $6 \mathrm{~d}$ of age and followed until $52 \mathrm{~d}$ on 2 commercial dairies. Calves were examined twice weekly by blinded members of the research team. Clinical respiratory and ultrasonographic lung scores were assigned at each exam and used to classify the first detected respiratory disease event (BRD1) into upper respiratory tract disease (clinical, no significant consolidation); subclinical lobular pneumonia (not clinical, patchy consolidation $\geq 1 \mathrm{~cm}^{2}$ ); clinical lobular pneumonia (clinical, patchy consolidation $\geq 1 \mathrm{~cm}^{2}$ ); subclinical lobar pneumonia (not clinical, $\geq 1$ lobe consolidated); and clinical lobar pneumonia (clinical, $\geq 1$ lobe consolidated). At BRD1, calves were blocked by their respiratory disease status and randomized to receive an antibiotic (tulathromycin, $2.5 \mathrm{mg} / \mathrm{kg}$ subcutaneous) or placebo (sterile saline, equal volume subcutaneous). Multivariable linear and logistic regression analyses were used to model response to therapy. At BRD1 ( $\mathrm{n}=289)$, the distribution of diagnoses was $29 \%$ (upper respiratory tract disease), $43 \%$ (subclinical lobular pneumonia), 13\% (clinical lobular pneumonia), $8 \%$ (subclinical lobar pneumonia), and $7 \%$ (clinical lobar pneumonia). Early antibiotic therapy limited progression of lung consolidation immediately following treatment, reduced the likelihood of requiring
\end{abstract}

\footnotetext{
Received December 11, 2019.

Accepted August 7, 2020.

*Corresponding author: ollivett@wisc.edu
}

treatment within $7 \mathrm{~d}$ of BRD1, and improved growth and mortality before weaning. Despite receiving multiple doses of antibiotics after BRD1, calves treated with either antibiotic or placebo were equally likely to enter the weaning phase with pneumonia. Clinical presentation was associated with response to treatment for worsening of consolidation, early treatment failure, days to retreatment, and average daily gain. Overall, treatment was associated with short-term benefits, but more research is needed to develop treatment protocols that more effectively treat pneumonia and ensure that calves enter the weaning period with ultrasonographically clean lungs.

Key words: bovine respiratory disease, antimicrobial stewardship, lung ultrasound, wean clean

\section{INTRODUCTION}

Respiratory disease is a leading cause of morbidity and mortality of preweaned dairy calves in the United States (USDA, 2018). According to producer-reported numbers, $12 \%$ of calves are treated for respiratory disease, with nearly one-quarter of preweaning deaths occurring subsequent to this condition (USDA, 2018). These reports likely underestimate the true prevalence of respiratory disease, as clinical presentation is inconsistent (Apley, 2014), producer diagnosis lacks sensitivity (Sivula et al., 1996; Cramer et al., 2016), and inapparent, subclinical pneumonia exists (Ollivett et al., 2015; Ollivett and Buczinski, 2016; Cramer and Ollivett, 2019).

In young dairy cattle, respiratory disease manifests most commonly as bronchopneumonia, an inflammatory condition of the lower airways secondary to bacterial infection. Bronchopneumonia is characterized by infiltration of neutrophils into the airspaces of the distal bronchioles and alveoli, which results in lung consolidation (Caswell, 2014). Lung ultrasound can be used to diagnose consolidation associated with 
this form of pneumonia. When combined with a clinical scoring system, portable lung ultrasound allows for the on-farm, antemortem diagnosis of upper respiratory tract disease, clinical pneumonia, and subclinical pneumonia (Ollivett et al., 2015; Ollivett and Buczinski, 2016; Cramer and Ollivett, 2019). Although lung ultrasound is not a perfect test, the sensitivity (79-94\%) and specificity (94-100\%) are high in dairy calves regardless of clinical status (Rabeling et al., 1998; Buczinski et al., 2015; Ollivett et al., 2015). The prevalence of clinical and subclinical lung consolidation is highly variable, ranging from 10 to $80 \%$, respectively (Ollivett and Buczinski, 2016; Buczinski et al., 2018; Cramer and Ollivett, 2019). In a cross-sectional study of Quebec dairy farms, ultrasonographic lung consolidation $\geq 3 \mathrm{~cm}$ was associated with season, group housing, and producer perception of a respiratory disease problem (Buczinski et al., 2018).

Given the high frequency at which respiratory disease can occur, it is clear that many dairy calves will suffer from the short- and long-term negative sequelae associated with respiratory disease. It has been established that clinical respiratory disease reduces welfare of calves (Mellor and Stafford, 2004), decreases growth (Stanton et al., 2012), increases the odds of leaving the herd before calving and during the first lactation (Bach, 2011; Schaffer et al., 2016), and is associated with a 233-kg loss in 305-d mature equivalent milk (Schaffer et al., 2016).

We have also learned that regardless of clinical status, 60-d-old calves with ultrasonographic lung consolidation were slower to get pregnant (Teixeira et al., 2017), 3-mo-old calves with lung consolidation were less likely to survive to first lactation (Adams and Buczinski, 2016), preweaned calves with at least $1 \mathrm{~cm}$ of ultrasonographic lung consolidation grew more slowly than unaffected calves (Cramer and Ollivett, 2019), and those with at least $3 \mathrm{~cm}$ of ultrasonographic lung consolidation produced $525 \mathrm{~kg}$ less $305-\mathrm{d}$ mature equivalent milk during their first lactation (Dunn et al., 2018) compared with unaffected herd mates.

Systemic antibiotic therapy is the mainstay of treatment for calves affected with bronchopneumonia. In theory, the earlier in the pathologic process that therapeutic drug levels are achieved within the parenchyma of the lung, the faster bacterial replication and neutrophil infiltration should cease, stifling the progression of lung lesions and hastening the resolution of lung consolidation. Therefore, the first objective of our randomized controlled study was to assess the effects of early antibiotic therapy on health and growth of preweaned dairy calves diagnosed with a new case of respiratory disease using clinical respiratory scoring and portable lung ultrasound. The second objective was to deter- mine whether treatment response depended on clinical presentation at the time of initial diagnosis. We hypothesized that early diagnosis and antibiotic therapy will improve ultrasonographic lung health, calf growth, and preweaning mortality compared with calves that experience a delay in antibiotic therapy.

\section{MATERIALS AND METHODS}

\section{Animals and Facilities}

Male and female Holstein calves, from 2 commercial dairy herds in southern Wisconsin, were enrolled into this randomized clinical trial and followed until weaning at approximately $56 \mathrm{~d}$ of age. Calves were fed 8 to $10 \mathrm{~L}$ of reconstituted milk replacer or pasteurized waste milk. Housing consisted of individual pens for the first 7 to $10 \mathrm{~d}$ of life, followed by group housing with automated feeders until weaned. This study was approved by the University of Wisconsin Institutional Animal Care and Use Committee (Madison, WI; Protocol \# V005250).

Calves were enrolled from December 2015 through May 2016, and follow-up extended through May 2016 for herd 1 and July 2016 for herd 2. Research staff visited each herd twice a week (Monday and Thursday or Tuesday and Friday) to enroll all available 3- to 5-d-old calves and perform follow-up examinations on all currently enrolled calves. At enrollment, research staff examined calves, measured heart girths, and collected whole blood samples. Once enrolled, every calf was subjected to twice-weekly (Monday and Thursday or Tuesday and Friday) examinations and heart girth measurements, as subsequently described, until they were weaned.

\section{Health Examinations and Data Collection}

All health examination data were recorded using the UW Calf Health Scorer iPad app (https://www.vetmed .wisc.edu/fapm/) and included a clinical respiratory examination, lung ultrasound, fecal score, and heart girth measurement on every enrolled calf at each visit. For the clinical respiratory exam, nasal discharge, ocular discharge, ear position, cough reflex, and rectal temperature were scored 0 to 3 , where $0=$ least affected and $3=$ most severely affected. Calves that scored $\geq 2$ in $\geq 2$ areas were considered positive for clinical respiratory disease (CRS+; McGuirk and Peek, 2014). Portable lung ultrasound (depth $9 \mathrm{~cm} ; 6.2-\mathrm{MHz}$ linear rectal transducer, Ibex Pro, E. I. Medical, Loveland, $\mathrm{CO})$ was used to assess the cranial-ventral aspects of the right and left lungs (Ollivett and Buczinski, 2016). More specifically, the following right lung lobes were 
Exam: USS, CRS, weight; eligible to be randomized to first treatment during twice weekly exams

Enrolled, collected blood for STP

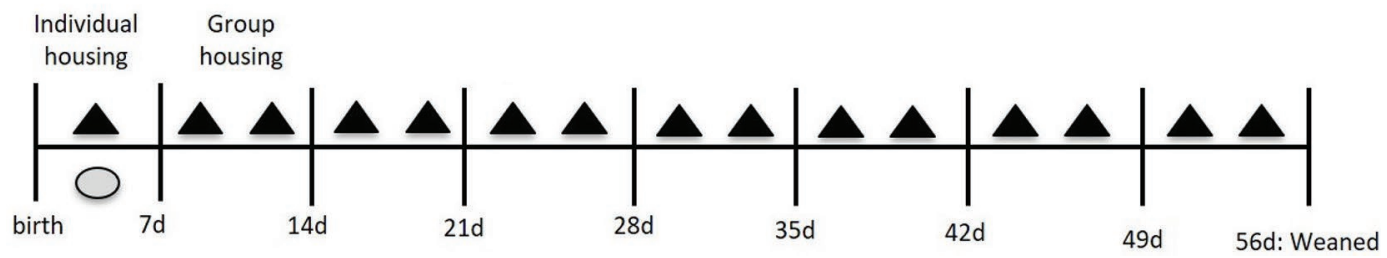

Figure 1. Timeline of enrollment and examinations throughout the 56-d study period. USS $=$ lung ultrasound score; CRS $=$ clinical respiratory score; STP = serum total protein.

systematically imaged: the cranial border of the caudal lung lobe in the sixth intercostal space (ICS), the right middle lung lobe in the fifth ICS, the caudal aspect of the cranial lung lobe in the fourth and third ICS, and the cranial aspect of the cranial lung lobe in the second and first ICS. The left lung lobes were also systematically imaged: the cranial border of the caudal lung lobe in the sixth ICS, the caudal aspect of the cranial lung lobe in the fifth and fourth ICS, and the cranial aspect of the cranial lung lobe in the third ICS.

Calves were assigned a lung ultrasound score (USS) that ranged from 0 to 5 , where $0=$ normal, aerated lung with reverberation artifact, $1=$ diffuse comet tails suggestive of pleural thickening, $2=$ lobular pneumonia with $\geq 1 \mathrm{~cm}^{2}$ consolidation, $3=$ lobar pneumonia with 1 lobe completely consolidated, $4=$ lobar pneumonia with 2 lobes completely consolidated, and $5=$ lobar pneumonia with $\geq 3$ lobes completely consolidated (Ollivett and Buczinski, 2016). Lobular pneumonia was defined as patchy areas of consolidation within an otherwise aerated lung lobe; the hyperechoic pleural interface with reverberation artifact of normal lung can be seen both dorsal and ventral to the lobular lesion when the probe is placed parallel to the rib within the ICS. Lobar pneumonia was defined by full-thickness consolidation of the lung lobe that extends proximally from the periphery of the lung lobe. Lobar lesions are hypoechoic and aerated lung is no longer visible ventral to the lesion (Ollivett and Buczinski, 2016).

Calf weights were estimated from heart girth measurements taken at each examination (https://extension .psu.edu/customized-dairy-heifer-growth-chart). At enrollment, blood was collected by jugular venipuncture using plastic 7-mL evacuated red-top tubes without additive (BD Vacutainer, Franklin Lakes, NJ). Samples were allowed to clot and separate at room temperature for $24 \mathrm{~h}$. Serum total protein (STP) concentration was then measured by digital refractometry to estimate transfer of maternal immunoglobulin (Misco
PA202X-003-105, Cleveland, OH). For the purpose of analysis, failure of passive transfer (FPT) was defined as STP $<5.5 \mathrm{~g} / \mathrm{dL}$ (Tyler et al., 1996).

Approximately $95 \%$ of all health scoring was performed by the first author (EB), with the last author (TO) making up the difference. TO provided training to EB before the start of the study and regularly monitored scoring throughout the study to ensure consistency and accuracy. Within approximately 1 min per calf, the respiratory score was performed, heart girth was measured, the lungs were scanned, and rectal temperature and fecal consistency were assessed, in that order. All exams took place within the individual or group pen, depending on the age of the calf (Figure 1).

\section{Randomization of Calves to Experimental Groups and Treatment Protocols}

Randomization decisions and follow-up treatment decisions, were based on the results of the current health examination, the previously generated allocation sequence, and the established protocols for secondand third-line treatments (Figure 2). These decisions, the calculation of drug dosages based on heart girth measurement, the administration of medications, and recording of treatments administered, were all done by the research team member responsible for restraining calves during the health examination. However, the research team member performing the health examinations, as well as farm staff, remained blinded to the randomization and treatment process. Blinding was maintained by administering treatments out of sight from the blinded individuals. In addition, the health examiner and farm staff did not have access to the allocation sequence or treatment records. To ensure food safety, on-farm treatment records were maintained by the research staff; however, specific drug names were not included. Instead, a generic "UW" comment was recorded in the calf's farm record, to indicate that 


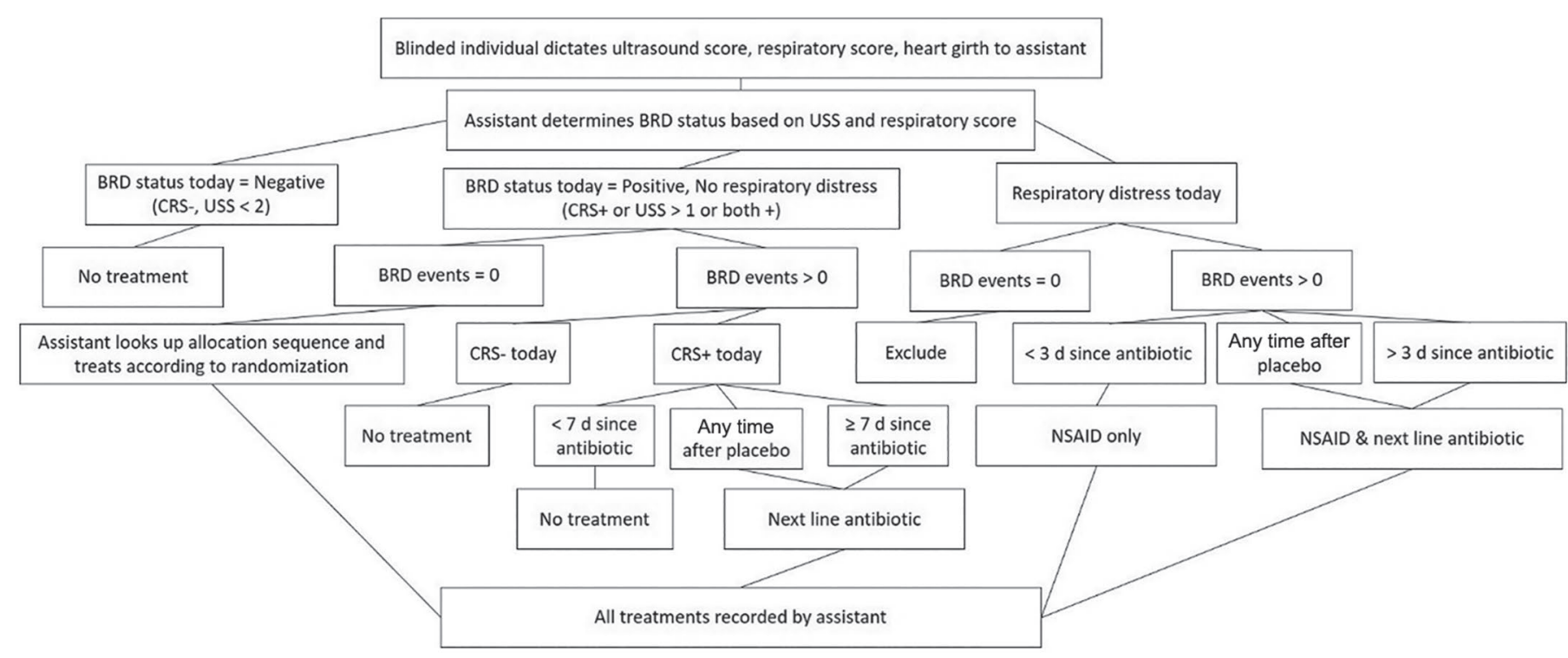

Figure 2. Flowchart describing decision making during each calf health examination. USS = lung ultrasound score; CRS $=$ clinical respiratory score; BRD = respiratory disease; NSAID = nonsteroidal anti-inflammatory (flunixin meglumine, $1.1 \mathrm{mg} / \mathrm{kg}$, intravenous).

either an antibiotic or placebo treatment had been administered. To further ensure blinding, a 60-d slaughter withhold was maintained for all randomized calves, even if they were randomized to the placebo group.

Calves were randomized to receive the first-line antibiotic [TX; tulathromycin (Draxxin, Zoetis, Parsippany, NJ), $2.5 \mathrm{mg} / \mathrm{kg}$, subcutaneous] or placebo $(\mathbf{C O N}$; equal volume of sterile saline, subcutaneous) when they tested positive for their first detected respiratory disease event (BRD1). For BRD1, a positive case was determined by combining a calf's clinical respiratory status with its lung ultrasound score, and included the following 5 potential categories of disease: upper respiratory tract infection (URT: CRS+, USS $<2$ ), subclinical lobular pneumonia [SLL: CRS negative $(\mathbf{C R S}-)$, USS $=2$ ]; clinical lobular pneumonia (CLL: CRS+, USS $=2$ ); subclinical lobar pneumonia (SL: CRS-, USS >2); and clinical lobar pneumonia (CL: CRS+, USS > 2; Ollivett and Buczinski, 2016; Cramer and Ollivett, 2019). The random allocation sequence incorporated blocking by herd and BRD1 status (e.g., URT, SLL, SL, CLL, and CL). The sequence was generated before the start of the study, using the random number function in Excel (Microsoft Corp., Redmond, WA), and remained concealed until each new case was assigned to a treatment group.

To provide a minimum standard of care, all calves affected by CL at BRD1 were administered 1 dose of a nonsteroidal anti-inflammatory (flunixin meglumine, $1.1 \mathrm{mg} / \mathrm{kg}$, intravenous). Calves exhibiting respiratory distress (RD; depressed, slow to stand or lie down, gaunt, new labored breathing, or open-mouthed breathing) at BRD1 were excluded from the study.

Second- and third-line treatments consisted of label doses of enrofloxacin and florfenicol, respectively, for calves with refractory clinical respiratory disease, using a standard 7-d post-treatment interval (PTI) as extrapolated from feedlot data (Apley, 2015). Exceptions to the standard PTI were made for calves originally diagnosed with subclinical disease (CRS-) at BRD1 and for those in RD following BRD1. More specifically, for subclinical calves randomized to the placebo group, the first-line antibiotic could be administered any time after BRD1 if they became CRS+ or developed RD. For clinical calves randomized to the placebo group, the first-line antibiotic could be administered at any point after BRD1 if they developed RD. If clinical calves that were randomized to the antibiotic group developed RD after BRD1, a nonsteroidal anti-inflammatory (flunixin meglumine, $1.1 \mathrm{mg} / \mathrm{kg}$, intravenous) was given, and the moratorium on giving the next line of antibiotic treatment was reduced from 7 to $3 \mathrm{~d}$.

In the absence of the research team, farm staff were instructed to observe and treat calves for clinical respiratory disease and record treatments according to the aforementioned treatment schedule after consulting with the last author (TO). Calves that were not detected with BRD1, that were treated by the farm at their first case of BRD, that were treated off-protocol at any time during the study, or that were treated for otitis media (abnormal ear position or head tilt as sole clinical abnormality) were excluded from analyses. 


\section{Additional Testing}

Sensitivity of bacterial respiratory pathogens to the respiratory antibiotics was confirmed through antimicrobial susceptibility testing (Supplemental Table S1, https://doi.org/10.3168/jds.2019-18044) at the Wisconsin Veterinary Diagnostic Laboratory (University of Wisconsin-Madison) using CLSI methods and interpretive criteria on deep nasal pharyngeal swabs from each herd before the start of the study. Salmonella newport was isolated from the maternity barn environment, maternity barn bedding, and a 7-d-old calf with bloody scours during the first month of data collection at herd 1.

\section{Statistical Analyses}

General Information. The experimental unit was the calf. A commercially available statistical package was used for all analyses (SAS version 9.4, SAS Institute Inc., Cary, NC). The sample size necessary to find a $0.07 \mathrm{~kg} / \mathrm{d}$ difference in ADG between TX and CON calves, assuming a standard deviation of 0.14 $\mathrm{kg} / \mathrm{d}$, power of 80 to $90 \%$, and $\alpha=0.05$, was 65 to 90 calves per treatment group, which included $4 \%$ increase to account for preweaning mortality (Ollivett, 2014). Intention-to-treat methods were used to prevent loss of observations from our data set due to missing values and to reduce potential bias that could occur from the exclusion of those calves. Specific details regarding imputation for missing values will be described later.

Outcomes. Outcomes were analyzed separately for each herd. Dichotomous outcomes were modeled using logistic regression and included worsening of ultrasonographic lung lesions at the exam following BRD1 (worse: $0=$ USS remained the same or decreased; 1 $=$ USS increased), early retreatment within $7 \mathrm{~d}$ of BRD1 (RETREAT: no =0; yes =1), completed 3-drug regimen of tulathromycin, enrofloxacin, and florfenicol (drug3: no $=0$; yes $=1$ ), respiratory disease at weaning [BRDW: $0=$ normal (CRS - , USS $<2$ ); $1=$ URT; 2 $=\mathrm{SLL} ; 3=\mathrm{CLL} ; 4=\mathrm{SL} ; 5=\mathrm{CL})$, and $\operatorname{died}(0=\operatorname{did}$ not die before $52 \mathrm{~d}$ of age; $1=$ died before $52 \mathrm{~d}$ of age). Continuous outcomes evaluated and modeled using linear regression included number of days to retreatment for clinical respiratory disease following treatment at BRD1 (modeled separately for subclinical and clinical BRD1 due to difference in retreatment protocol) and ADG $(\mathrm{kg} / \mathrm{d})$.

Explanatory Variables. The primary explanatory variable of interest was treatment group $(\mathrm{CON}=0$; $\mathrm{TX}$ $=1$ ). Other variables assessed as potential confounding variables included sex $(0=$ female; $1=$ male $)$, dystocia $(0=$ no assisted delivery required; $1=$ assisted delivery required $), \operatorname{FPT}(0=\mathrm{STP} \geq 5.5 \mathrm{~g} / \mathrm{dL} ; 1=\mathrm{STP}<5.5$ $\mathrm{g} / \mathrm{dL})$, scours during first $3 \mathrm{wk}$ of life $(0=\mathrm{FS}<3 ; 1=$ FS 3), other diseases (navel or joint score only $<2=0$; navel or joint score $\geq 2$ ever $=1)$; season of birth $(1=$ Dec-Feb; 2 = Mar-Apr; 3 = May-Jul), BRD1 (URT, SLL, SL, CLL, and CL), age at BRD1 (below median age at first treatment $=0$; above median age at first treatment $=1$ ), and weight1 (below median enrollment weight $=0$; above median enrollment weight $=1$ ). Raw comparisons of categorical risk factors were evaluated using chi-square analysis or Fisher's exact test (if expected cell count was $<5$ ) and included sex, dystocia, FPT, scours, and other diseases. Raw comparisons of continuous risk factors were evaluated using Wilcoxon rank-sum [enrollment age; median (interquartile range)] and $t$-test procedures [enrollment weight and raw ADG; mean (SD)].

Testing Univariable and Multivariable Associations. Univariable analysis of explanatory variables that resulted in $P \leq 0.20$ were included as fixed effects in the multivariable model. Treatment and BRD1 status were forced into the full multivariable model regardless of significance at the univariable level, but only the primary predictor of interest (treatment) was forced into each final model. Two-way interactions between treatment and the potential confounding variables described previously were tested for significance. Manual backward stepwise elimination was used to refine the model until all remaining variables were significant at the $\alpha \leq 0.05$ level in the type 3 tests of fixed effects. To assess for confounding, a criterion of $\geq 20 \%$ change in estimate for treatment was used before final elimination of a variable. The CON group was chosen as the reference category for treatment group in all models. The LSMEANS statement with the DIFF option was used to compare predicted means, and Tukey-Kramer was used to adjust for multiple comparisons. For linear regression models, the final model was assessed graphically for outliers, influential observations, and normality, using plots of fitted values versus Studentized residuals, Cook's distance, and leverage. Tests of normality (Shapiro-Wilk, Kolmogorov-Smirnov, Cramer-von Mises, or Anderson-Darling) were performed for continuous variables. Significance was declared at $\alpha \leq 0.05$. Missing values for FPT and dystocia were imputed as the null $(=0)$, whereas missing enrollment weights were imputed as the average for the group, and the last observation carried forward was used for BRDW and ADG.

Model 1: Worsening of Lung Lesions. Calves scoring USS $=5$ at BRD1 (SL: $\mathrm{n}=2$; CL: $\mathrm{n}=3$ ) could not develop a higher score and were excluded from the worsening of lung lesions analysis. Additionally, calves that died before their health exam following BRD1 
were also excluded $(\mathrm{n}=7)$. The primary predictor of interest was treatment. The hypothesis was that treatment would be negatively associated with lung lesion worsening. For herd 1, the final multivariable model included treatment group and BRD1. For herd 2, none of the predictor variables were significant in the final model, although treatment group remained, as it was forced into all final models.

Model 2: Retreatment Within 7 Days of BRD1. Calves that died within $7 \mathrm{~d}$ of BRD1 that were not retreated were excluded for analysis of RETREAT. The predictor of interest was treatment. The hypothesis was that treatment group would be associated with retreatment within $7 \mathrm{~d}$ of BRD1. For herd 1, the final multivariable model included treatment group, weight1, and BRD1. For herd 2, the final multivariable model included treatment group only.

Model 3: Days to Retreatment for Calves with Subclinical BRD1. Calves that were subclinical at BRD1 (SLL and SL; $\mathrm{n}=148$ ) and were later treated for clinical respiratory disease were included in this analysis (retreated; $\mathrm{n}=118$ ). Days to retreatment were not normally distributed and were transformed to natural $\log$ for the univariable analysis and multivariable modeling. The predictor of interest was treatment; BRD1 status (SLL and SL) was included as a fixed effect. The hypothesis was that treatment was associated with days to retreatment. For herd 1, the final multivariable model included treatment group and BRD1. For herd 2, the final multivariable model included treatment group and season. Results are reported as back-transformed means \pm standard error of the mean (SEM).

Model 4: Days to Retreatment for Calves with Clinical BRD1. Calves that were clinical at BRD1 (URT, CLL, and CL; n = 141) and were later treated for continued or additional clinical respiratory disease were included in this analysis (retreated; $\mathrm{n}=109$ ). As in the previous analysis, days to retreatment were not normally distributed and were transformed to natural $\log$ for the univariable analysis and multivariable modeling. The predictor of interest was treatment; BRD1 status (URT, CLL, and CL) was included as a fixed effect. The hypothesis was that treatment was associated with days to retreatment. For herd 1, the final multivariable model included treatment group. For herd 2 , the final multivariable model included treatment group and age at first treatment. Results are reported as back-transformed means \pm SEM.

Model 5: Completing Full Antibiotic Treatment Protocol. The predictor of interest was treatment. The hypothesis was that treatment was associated with completion of the full antibiotic treatment protocol. For herd 1, the final multivariable model included treatment group and age at first treatment. For herd 2, the final multivariable model included group and age at first treatment.

Model 6: Respiratory Disease at Weaning $(B R D W)$. The predictor of interest was treatment. The hypothesis was that treatment was associated with respiratory disease status at weaning. For calves that died before $52 \mathrm{~d}$ of age, their BRD1 status at last observation was carried forward for the purpose of analysis. For herd 1, the final multivariable model included treatment group only. For herd 2, the final multivariable model included treatment group and season.

Model 7: Mortality. When modeling the probability of death, the predictor of interest was treatment. The hypothesis was that treatment was associated with the odds of death. For herd 1, the final multivariable model included treatment group, FPT, and age at first treatment. Only 1 death occurred for herd 2; therefore mortality was not modeled as an outcome.

Model 8: $A D G$. Average daily gain for each calf was calculated from individual measurements (REG procedure), where weight at each exam was the dependent variable and age at each exam was the independent variable. The coefficient for age $(\mathrm{kg} / \mathrm{d})$ represented the change in weight over time and was considered the ADG. The primary predictor of interest was treatment. The hypothesis was that the effect of treatment on ADG would depend on BRD1 status. For herd 1, the final multivariable model included treatment group, BRD1, FPT, sex, season, and the interaction between treatment group and season. For herd 2, the final multivariable model included treatment group and BRD1.

\section{RESULTS}

\section{Herd Characteristics}

A total of 357 calves were initially enrolled into the study. Calves were enrolled at $4(4,5)$ and $5(3,6) \mathrm{d}$ of age for herd 1 and herd 2, respectively. First detected respiratory disease event, and therefore allocation to treatment group, occurred overall at $12(8,18) \mathrm{d}$ of age. More specifically, BRD1 occurred at $11(8,16)$ and 15 $(10,22) \mathrm{d}$ of age for herd 1 and herd 2, respectively. Sixty-eight calves were excluded from analyses for the following reasons: no respiratory disease $(\mathrm{n}=30)$, protocol deviation $(\mathrm{n}=19)$, and respiratory distress at BRD1 ( $\mathrm{n}=19)$. Table 1 describes the distribution of calf characteristics by treatment group in each herd for the remaining 289 calves. Of the 289 calves that developed respiratory disease, $51 \%(\mathrm{n}=148)$ had subclinical respiratory disease, indicating a nearly equal ratio of clinical to subclinical calves at BRD1. Additionally, $17 \%$ of calves $(50 / 289)$ died or were euthanized by 52 $\mathrm{d}$ of age. Of those 50 calves, 49 were enrolled at herd 1. 
Calf-level data were missing and therefore imputed for dystocia $(\mathrm{n}=11), \operatorname{FPT}(\mathrm{n}=7)$, and enrollment weight $(\mathrm{n}=10)$.

\section{Change in Ultrasonographic Lung Lesions Between Treatment and the Following Examination}

Overall, ultrasound scores were higher at the exam following treatment for BRD1 in $23 \%(\mathrm{n}=35)$ of $\mathrm{TX}$ and $38 \%(\mathrm{n}=50)$ of CON calves $(P<0.01)$. The predicted mean probabilities of having a higher USS at the exam following BRD1 for each herd are shown in Table 2 . In herd 1 , the CON calves were twice as likely to have a worse lung USS compared with TX calves. A similar numerical pattern was observed for herd 2 . The manner in which respiratory disease presented at BRD1 was retained as a significant predictor of worsening lung lesions only in herd 1 . For this herd, relative to calves with subclinical lobular lesions, clinical calves with $1 \mathrm{~cm}$ or more of patchy lung lesions (CLL) were approximately 4 times more likely to develop more extensive lung lesions between BRD1 and the following examination [odds ratio (OR): 4.2; $95 \%$ confidence interval (CI): 1.13, 16.0].

\section{Probability of Retreatment Within $7 d$ of BRD1}

Twenty-one percent $(31 / 147)$ of TX and $48 \%$ $(60 / 125)$ of CON calves required retreatment within $7 \mathrm{~d}$ of BRD1 $(P<0.0001)$. For both herds, compared with TX calves, CON calves were approximately 4.5 times more likely to be retreated within $7 \mathrm{~d}$ following BRD1 after multivariable analysis (Table 2). At herd 1 , subclinical lobular calves were significantly less likely to require retreatment compared with clinical lobar calves (OR: 0.16 ; $95 \%$ CI: 0.03 , 0.96; adjusted $P$ value $=0.04)$. However, the manner in which BRD initially presented was not associated with retreatment within $7 \mathrm{~d}$ at herd 2.

\section{Days Until Retreatment After BRD1}

A total of 148 calves were diagnosed with subclinical respiratory disease at BRD1 (TX: $\mathrm{n}=76$; CON: $\mathrm{n}=72)$. Eighty percent $(\mathrm{n}=61)$ of subclinical TX calves and $79 \%(\mathrm{n}=57)$ of subclinical CON calves were retreated during the follow-up period. After multivariable analyses of each herd, TX calves were retreated for clinical disease significantly later than CON calves (Table 3). At herd 1, in calves diagnosed with subclinical pneumonia, those with lobular (SLL) lesions were retreated later than calves with lobar (SL) lesions (SLL: $11.8 \pm 1.12 \mathrm{~d}$; SL: $4.2 \pm 1.20 \mathrm{~d} ; P=0.02$ ).
A total of 141 calves were diagnosed with clinical respiratory disease at BRD1 (TX: $\mathrm{n}=76$; CON: $\mathrm{n}=$ $65)$. Seventy-five percent $(\mathrm{n}=57)$ of clinical TX calves and $80 \%(\mathrm{n}=52)$ of clinical CON calves were retreated during the follow-up period. For both herds, TX calves were retreated significantly later after BRD1 compared with CON calves (Table 3). The amount of lung consolidation present at BRD1 in clinical calves was not associated with days to retreatment for either herd.

\section{Completing the Full Antibiotic Protocol and Respiratory Disease at Weaning}

Of the 239 calves that survived to weaning, similar proportions of TX and CON calves completed the full antibiotic protocol [TX: $65.9 \%$ (87/132); CON: $66.4 \%$ $(71 / 107) ; P=0.94]$ and had lung consolidation at weaning [TX: 81\% (107/132); CON: 80\% (86/107); $P$ $=0.89]$. Of the abnormal calves at weaning, pneumonia was subclinical in $92 \%(98 / 107)$ and $86 \%(75 / 87)$ of TX and CON calves, respectively $(P=0.29)$. After multivariable analyses for each herd using intention-totreat methods, TX calves were just as likely as CON calves to complete the full treatment protocol (Table 2 ). Similarly, TX calves were just as likely as CON calves to have lung consolidation at weaning (Table 2).

\section{Mortality}

Of the 289 calves included in the analysis, 50 (17\%) died before weaning, with 49 out of 50 occurring in calves from herd 1 . Thirteen percent (20/152) of TX calves died, compared with $22 \%(30 / 137)$ of CON $(P$ $=0.05)$. In herd 1 , where most deaths occurred, CON calves were twice as likely to die by the end of the study period compared with TX calves, based on the final multivariable model (Table 2).

\section{Calf Growth}

The raw ADG was $0.65 \pm 0.03 \mathrm{~kg} / \mathrm{d}$ for TX and 0.52 $\pm 0.03 \mathrm{~kg} / \mathrm{d}$ for CON $(\mathrm{n}=289 ; P<0.001)$. The raw ADG for each BRD1 status was $0.61 \pm 0.03 \mathrm{~kg} / \mathrm{d}$ (SLL), $0.62 \pm 0.04 \mathrm{~kg} / \mathrm{d}(\mathrm{URT}), 0.49 \pm 0.05 \mathrm{~kg} / \mathrm{d}$ (CLL), 0.63 $\pm 0.07 \mathrm{~kg} / \mathrm{d}(\mathrm{SL})$, and $0.49 \pm 0.07 \mathrm{~kg} / \mathrm{d}(\mathrm{CL} ; P=$ 0.10). After fitting multivariable models for each herd, TX calves still grew faster than CON calves; however, in herd 1, the effect on ADG was present only during the coldest months, December and January (Table 3). For both herds, calves with SL at BRD1 had the highest numerical ADG (Table 4); however, it was only at herd 2 that calves with SL at BRD1 grew significantly faster compared with CLL after accounting for multiple comparisons $(P=0.03$; Table 4$)$. 
Binversie et al.: EFFECT OF ANTIBIOTICS ON CALF HEALTH AND GROWTH

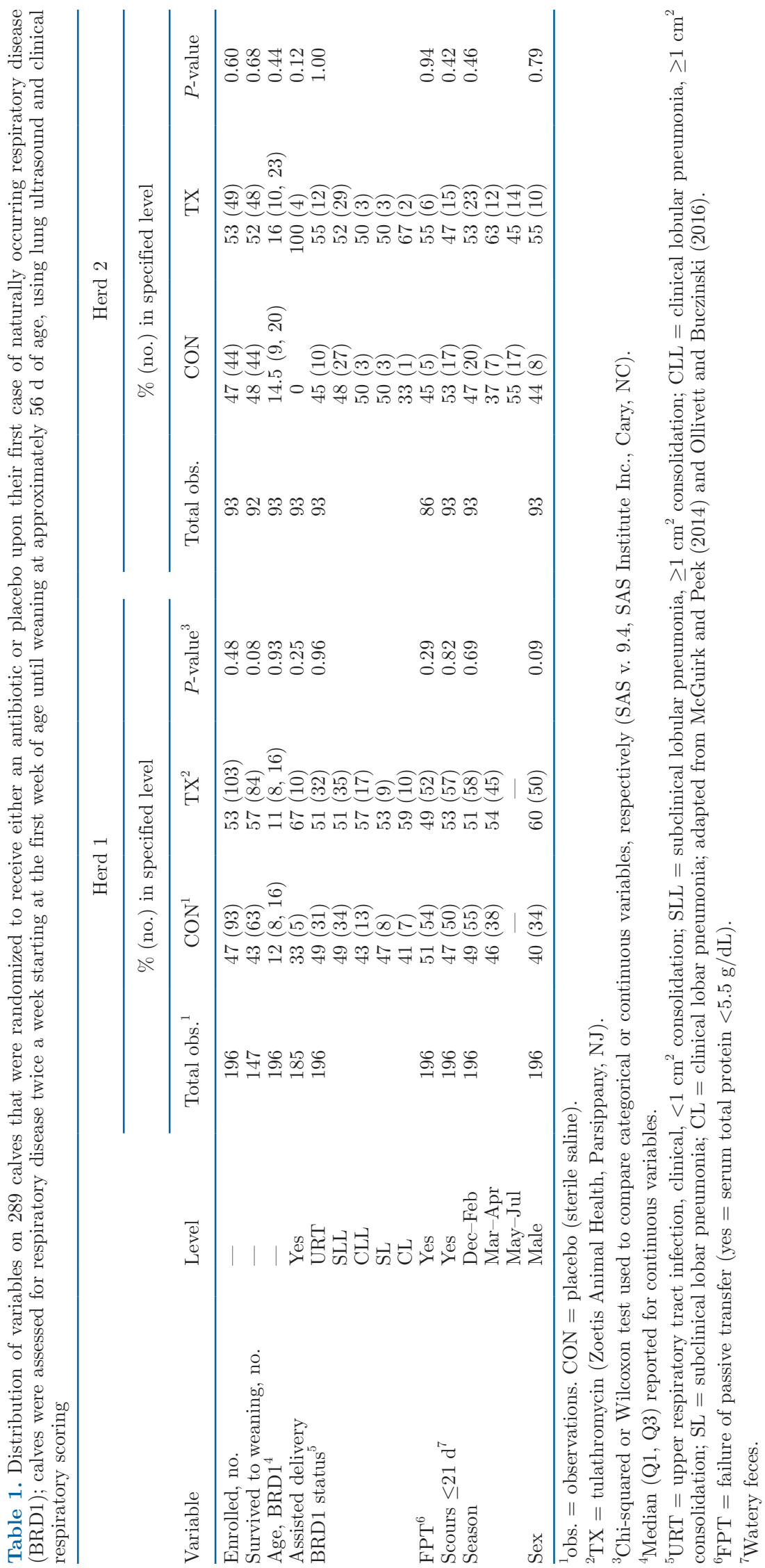




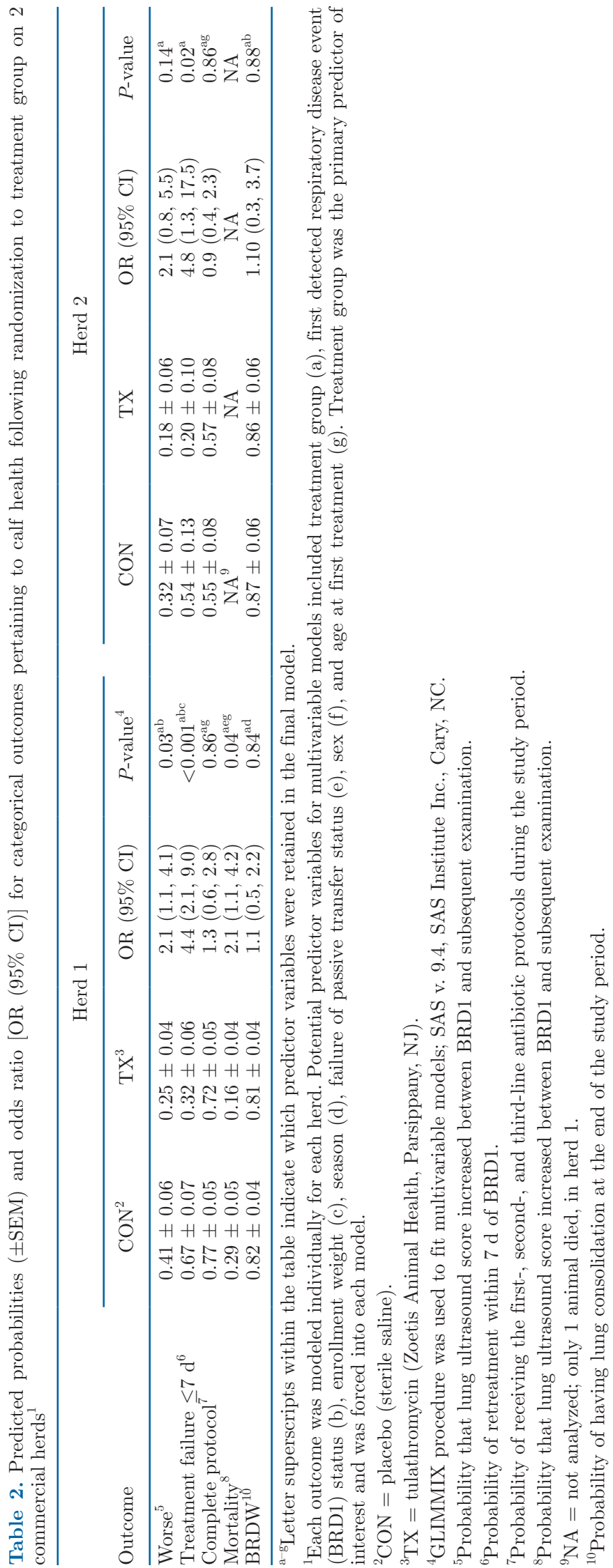


Table 3. Predicted means $( \pm \mathrm{SEM})$ for continuous outcomes pertaining to calf health following randomization to treatment group after a diagnosis of naturally occurring respiratory disease on 2 commercial dairy herds ${ }^{1}$

\begin{tabular}{|c|c|c|c|c|c|c|}
\hline Outcome & \multicolumn{3}{|c|}{ Herd $1(\mathrm{n}=196)$} & \multicolumn{3}{|c|}{ Herd $2(\mathrm{n}=93)$} \\
\hline $\begin{array}{l}\text { Time to clinical, }{ }^{5} \mathrm{~d} \\
\text { Time to re-treat, }{ }^{6} \mathrm{~d} \\
\text { ADG }^{7} \\
(\text { Group } \times \text { season) Dec-Jan } \\
\text { Feb-Mar }\end{array}$ & $\begin{array}{c}6.8 \pm 1.1 \\
6.9 \pm 1.1 \\
0.38 \pm 0.04 \\
0.53 \pm 0.04\end{array}$ & $\begin{array}{l}11.8 \pm 1.1 \\
11.5 \pm 1.1 \\
0.59 \pm 0.03 \\
0.52 \pm 0.04\end{array}$ & $\begin{aligned} & 0.001^{\mathrm{ab}} \\
< & 0.0001^{\mathrm{a}} \\
< & 0.0001^{\text {abdef }} \\
& 0.88^{\text {abdef }}\end{aligned}$ & $\begin{array}{c}11.2 \pm 1.2 \\
5.8 \pm 1.2 \\
0.79 \pm 0.03\end{array}$ & $\begin{aligned} 15.5 & \pm 1.2 \\
9.9 & \pm 1.1 \\
0.87 & \pm 0.03\end{aligned}$ & $\begin{array}{l}0.04^{\mathrm{ad}} \\
0.005^{\mathrm{ag}} \\
0.04^{\mathrm{ab}}\end{array}$ \\
\hline
\end{tabular}

\footnotetext{
${ }^{\mathrm{a}-\mathrm{g}}$ Letter superscripts within the table indicate which predictor variables were retained in the final model.

${ }^{1}$ Each outcome was modeled individually for each herd. Potential predictor variables for multivariable models included treatment group (a), first detected respiratory disease event (BRD1) status (b), enrollment weight (c), season (d), failure of passive transfer status (e), sex (f), and age at first treatment $(\mathrm{g})$. Treatment group was the primary predictor of interest and was forced into each model.

${ }^{2} \mathrm{CON}=$ placebo (sterile saline).

${ }^{3} \mathrm{TX}=$ tulathromycin (Zoetis Animal Health, Parsippany, NJ).

${ }^{4}$ MIXED procedure was used to fit multivariable models for continuous outcomes; SAS v. 9.4, SAS Institute Inc., Cary, NC.

${ }^{5}$ Subclinical calves only.

${ }^{6}$ Clinical calves only.

${ }^{7}$ Group $\times$ season interaction was present for herd 1 only.
}

\section{DISCUSSION}

To the authors' knowledge, this is the first randomized controlled field trial to measure the effect of early antibiotic therapy on incident (new) cases of naturally occurring respiratory disease that were detected using a combination of portable lung ultrasound and clinical respiratory scoring. Antibiotic therapy using a single dose of a long-acting macrolide appeared to delay the progression of lung consolidation and the need for retreatment, reduce mortality, and increase growth under some circumstances. We also found that the manner in which respiratory disease initially manifested determined how calves performed for certain outcomes, including worsening of consolidation, early treatment failure, days to clinical disease for calves that were initially subclinical, and ADG, although these findings were herd-specific. Although the herds enrolled in this study used similar general management and housing practices, we suspect that higher-quality nutrition (pasteurized waste milk vs. milk replacer), lower stocking densities, and lower pathogen challenges in herd 2 might be driving the difference in outcomes, especially regarding mortality and ADG. However, genetic differ-

Table 4. Predicted means $( \pm \mathrm{SEM})$ for outcomes pertaining to calf health following randomization to treatment group after a diagnosis of naturally occurring respiratory disease on 2 commercial dairy herds ${ }^{1}$

\begin{tabular}{|c|c|c|c|c|c|c|}
\hline Outcome & Herd & $\mathrm{SLL}^{2}$ & \multicolumn{4}{|c|}{ BRD1 status } \\
\hline Worse $^{7}$ & $1^{\mathrm{ab}}$ & $0.20 \pm 0.05$ & $0.36 \pm 0.06$ & $0.51 \pm 0.09$ & $0.29 \pm 0.11$ & $0.30 \pm 0.11$ \\
\hline Time to clinical, ${ }^{9} \mathrm{~d}$ & $1^{\mathrm{ab}}$ & $11.8 \pm 1.12$ & - & - & $4.2 \pm 1.20$ & - \\
\hline $\mathrm{ADG}, \mathrm{kg} / \mathrm{d}$ & $1^{\text {abdef }}$ & $0.48 \pm 0.03$ & $0.57 \pm 0.03$ & $0.44 \pm 0.05$ & $0.58 \pm 0.06$ & $0.45 \pm 0.06$ \\
\hline $\mathrm{ADG}, \mathrm{kg} / \mathrm{d}$ & $2^{\mathrm{ab}}$ & $0.84 \pm 0.03$ & $0.82 \pm 0.03$ & $0.73 \pm 0.05$ & $1.01 \pm 0.06$ & $0.76 \pm 0.06$ \\
\hline
\end{tabular}

${ }^{\mathrm{a}-\mathrm{g}}$ Letter superscripts within the table indicate which predictor variables were retained in the final model.

${ }^{1}$ Outcomes were modeled individually for each herd and only included in the table if first detected respiratory disease event (BRD1) status was statistically significant $(P \leq 0.05)$ and therefore retained in the final model. Potential predictor variables for multivariable models included treatment group (a), BRD1 status (b), enrollment weight (c), season (d), failure of passive transfer status (e), sex (f), and age at first treatment (g). ${ }^{2} \mathrm{SLL}=$ subclinical lobular pneumonia, $\geq 1 \mathrm{~cm}^{2}$ consolidation.

${ }^{3} \mathrm{URT}=$ upper respiratory tract infection, clinical, $<1 \mathrm{~cm}^{2}$ consolidation.

${ }^{4} \mathrm{CLL}=$ clinical lobular pneumonia, $\geq 1 \mathrm{~cm}^{2}$ consolidation.

${ }^{5} \mathrm{SL}=$ subclinical lobar pneumonia.

${ }^{6} \mathrm{CL}=$ clinical lobar pneumonia. Disease definitions adapted from McGuirk and Peek (2014) and Ollivett and Buczinski (2016).

${ }^{7}$ Probability that lung ultrasound score increased between BRD1 and the subsequent examination.

${ }^{8}$ Probability of retreatment within 7 d of BRD1.

${ }^{9}$ Days until clinical for calves with subclinical disease at BRD1. 
ences could also be contributing, for ADG in particular, and cannot be ruled out.

Many young dairy calves develop respiratory disease early in life and face the stressful weaning period with ultrasonographically consolidated lungs. In the current study, more than $75 \%$ of the calves enrolled during the 6-mo period developed respiratory disease. Half of the BRD1 cases occurred between 8 and $18 \mathrm{~d}$ of age, and just over $70 \%$ still had lobar pneumonia at weaning even though most calves were treated 2 to 3 times. In terms of external validity, readers should be aware that a similar study design implemented on a dairy with lower disease pressure might yield different results.

Given the high proportion of affected calves within a relatively narrow management window of 2 and $3 \mathrm{wk}$ of age, some dairy producers and veterinarians might consider the use of targeted metaphylaxis in this scenario. Although substantial clinical data exists regarding the potential benefit of metaphylaxis, little to no data exists regarding the benefit of group treatment in preweaned dairy calves when lung ultrasound is used to determine timing of the intervention as well as to assess lung health outcomes. Compared with individual detection and treatment programs, it is possible that metaphylaxis might result in fewer treatment failures, less subsequent antibiotic usage, and greater likelihood of weaning with ultrasonographically clean lungs. However, well-designed on-farm studies are needed that incorporate ultrasound to assess lung health at weaning to confirm success and utility of metaphylaxis in young dairy calves. Even if metaphylaxis improves treatment response and lung health, any underlying management deficits would still need to be corrected to reduce the high-risk characteristics of calves and the need for metaphylaxis.

In our study, individual calves were intensively screened twice a week with respiratory scoring and lung ultrasound to initiate treatment as close to the onset of incident, or new, cases of disease as possible. Our use of the Wisconsin calf health scorer app was intended to help keep clinical respiratory scoring and lung scoring consistent by providing the same definition for clinical disease for all calves. Because we used a very rigid definition of clinical disease (2 categories scoring 2 or more; McGuirk and Peek, 2014; Cramer and Ollivett, 2019), it is possible that some calves were not included in the clinical category. One argument against using the Wisconsin score to define clinical disease is the absence of attitude assessment and the characteristics of respiration (rate, effort). It is possible that a calf exhibiting increased expiratory effort would be characterized as subclinical if the only CRS abnormality was a mild nasal discharge. However, each calf was simultaneously assessed for respiratory distress, as previously described in this report, and excluded from analysis $(\mathrm{n}=19)$. The Wisconsin score does lack sensitivity (Buczinski et al., 2015) and will miss some calves with respiratory disease, which was the rationale for including lung ultrasound. Lung ultrasound is very sensitive for detecting the lung lesions associated with respiratory disease in calves that are not positive based on the Wisconsin score (Ollivett et al., 2015). We defined ultrasonographic pneumonia as the presence of at least $1 \mathrm{~cm}$ of lung lesion and subsequently categorized each case based on the number of lobes that were completely consolidated (lobar pneumonia). Data recently reported by our group has shown that calves with at least $1 \mathrm{~cm}^{2}$ of lesion grow more slowly than unaffected calves (Cramer and Ollivett, 2019). Thus, although our method of diagnosis is not considered a gold standard, it likely represents the closest, most practical way that we have at the moment to characterize consequential lung disease in dairy calves.

It is also conceivable that these results are specific to the 2 participating commercial dairies; however, our group has reported similar lung level outcomes under a controlled setting involving experimentally induced pneumonia in young dairy calves (Holschbach et al., 2019). In that study, although a 3-d course of injectable ampicillin resulted in rapid improvement of ultrasonographic lung lesions in nearly all calves after experimental challenge with Pasteurella multocida, the lung lesions recurred within a week after treatment and persisted until the end of the study despite relatively normal clinical exams. In addition to the relapse of lung lesions, Pasteurella multocida could be grown from the lung tissue at necropsy $14 \mathrm{~d}$ after treatment, indicating that the resolution of clinical signs was not associated with a simultaneous bacteriological cure.

For our current study, antibiotics were selected based on farm preference, ease of administration, adherence to a 7-d PTI, and sensitivity patterns of previously detected respiratory pathogens on each farm. Although our susceptibility testing suggested that the antimicrobials used in this study should be effective, it is possible that in vivo efficacy did not mimic in vitro efficacy. Tulathromycin, the first-line drug, is a macrolide antibiotic that functions by interrupting protein synthesis in a broad array of bacterial and mycoplasma species. Researchers have discussed how to measure the relative benefits of antibiotic treatment and explained the theory behind PTI (Apley, 2015; DeDonder and Apley, 2015). However, no current consensus in the dairy industry exists on how best to treat calves affected by respiratory disease, and label dosing strategies are based on vague clinical definitions of treatment success (e.g., survival, temperature $<40^{\circ} \mathrm{C}$, normal attitude, normal respiratory pattern; DeDonder and Apley, 2015). 
Conversely, treatment guidelines have been developed and implemented for treating humans with communityacquired pneumonia since the late 1990s (as reviewed by Bender and Niederman, 2018). Interestingly, in the United States, current recommendations are to treat empirically for "atypical pathogens" (Mycoplasma, Chlamydia, and Legionella) using a macrolide antibiotic, and to include a $\beta$-lactam to provide coverage against streptococcal species; or to consider monotherapy with a fluoroquinolone (Bender and Niederman, 2018). Based on patient mortality outcomes, macrolide monotherapy is strongly discouraged unless the patient is a young adult without comorbidities, immunocompromise, recent antibiotic treatment within the past 3 mo, or other stressors (Bender and Niederman, 2018). Young, preweaned dairy calves are exposed to social stressors, environmental challenges, and common comorbidities (e.g., diarrhea), particularly when housed in large group settings. Although respiratory pathogens differ in some ways between humans and cattle, it is unlikely that young dairy calves would meet the conditions for macrolide monotherapy in the context of human medicine and desired outcomes. More research is needed to understand whether treatment response and resolution of lung consolidation can be improved through combination therapies.

Last, although producers were encouraged to visually screen calves for illness and record treatments for the $3 \mathrm{~d} /$ wk that research staff was not on farm, we cannot dismiss the possibility that treatment of some calves could have been inadvertently delayed because farm staff knew that we would visit the next day. Measures were taken to minimize this risk, including clearly outlining expectations before the start of the study, easy-to-implement treatment logs, and regular communication between farm and research staff via phone and email; the study coordinator was readily available for phone consultation on weekends and throughout the week.

\section{CONCLUSIONS}

This study demonstrated several short-term benefits of early antibiotic therapy after using respiratory scoring and lung ultrasound to diagnose respiratory disease. Unfortunately, this study also highlighted that lung consolidation can recur and persist to weaning despite early diagnosis, multiple antibiotic treatments, and resolution of clinical disease. Although this discussion is not intended to be a referendum on the value of a single drug, it should serve to stimulate more thought and research on how we address antimicrobial stewardship and quantify treatment success of antibiotic protocols on farms and in research settings.

\section{ACKNOWLEDGMENTS}

This study was supported from funding received from the American Association of Bovine Practitioners (AABP; Ashland, OH) Foundation Grant. The authors thank the dairy producers for allowing us to perform research and student volunteers (University of Wisconsin, Madison) for their assistance with data collection. The authors have not stated any conflicts of interest.

\section{REFERENCES}

Adams, E. A., and S. Buczinski. 2016. Short communication: Ultrasonographic assessment of lung consolidation postweaning and survival to the first lactation in dairy heifers. J. Dairy Sci. 99:14651470 .

Apley, M. 2014. The clinical syndrome of BRD: What it is and what it is not. Anim. Health Res. Rev. 15:135-137. https://doi.org/10 $.1017 /$ S1466252314000152.

Apley, M. 2015. Treatment of calves with bovine respiratory disease: Duration of therapy and posttreatment intervals. Vet. Clin. North Am. Food Anim. Pract. 31:441-453. https://doi.org/10.1016/j .cvfa.2015.06.001.

Bach, A. 2011. Associations between several aspects of heifer development and dairy cow survivability to second lactation. J. Dairy Sci. 94:1052-1057. https://doi.org/10.3168/jds.2010-3633.

Bender, M. T., and M. S. Niederman. 2018. Treatment guidelines for community-acquired pneumonia. Ann. Res. Hosp. 2:6. https://doi .org/10.21037/arh.2018.05.01.

Buczinski, S., M. E. Borris, and J. Dubuc. Herd-level prevalence of the ultrasonographic lung lesions associated with bovine respiratory disease and related environmental risk factors. 2018. J. Dairy Sci. 101:2423-2432. https://doi.org/10.3168/jds.2017-13459.

Buczinski, S., T. L. Ollivett, and N. Dendukuri. 2015. Short communication: Bayesian estimation of the accuracy of the calf respiratory scoring chart and ultrasonography for the diagnosis of bovine respiratory disease in pre-weaned dairy calves. Prev. Vet. Med. 119:227-231. https://doi.org/10.1016/j.prevetmed.2015.02.018.

Caswell, J. L. 2014. Failure of respiratory defenses in the pathogenesis of bacterial pneumonia of cattle. Vet. Pathol. 51:393-409. https:// doi.org/10.1177/0300985813502821.

Cramer, M. C., and T. L. Ollivett. 2019. Growth of preweaned, grouphoused dairy calves diagnosed with respiratory disease using clinical respiratory scoring and thoracic ultrasound-A cohort study. J. Dairy Sci. 102:4322-4331. https://doi.org/10.3168/jds.2018-15420.

Cramer, M. C., T. L. Ollivett, and A. L. Stanton. 2016. Associations of behavior-based measurements and clinical disease in preweaned, group-housed dairy calves. J. Dairy Sci. 99:7434-7443. https://doi .org/10.3168/jds.2015-10207.

DeDonder, K. D., and M. D. Apley. 2015. A review of the expected effects of antimicrobials in bovine respiratory disease treatment and control using outcomes from published randomized clinical trials with negative controls. Vet. Clin. North Am. Food Anim. Pract. 31:97-111. https://doi.org/10.1016/j.cvfa.2014.11.003.

Dunn, T. R., T. L. Ollivett, D. L. Renaud, K. E. Leslie, S. J. LeBlanc, T. F. Duffield, and D. F. Kelton. 2018. The effect of lung consolidation, as determined by ultrasonography, on first lactation milk production in Holstein dairy calves. J. Dairy Sci. 101:5404-5410. https://doi.org/10.3168/jds.2017-13870.

Holschbach, C. L., S. M. Raabis, and T. L. Ollivett. 2019. Effect of antibiotic treatment in preweaned Holstein calves after experimental bacterial challenge with Pasteurella multocida. J. Dairy Sci. 102:11359-11369. https://doi.org/10.3168/jds.2019-16992.

McGuirk, S. M., and S. F. Peek. 2014. Timely diagnosis of dairy calf respiratory disease using a standardized scoring system. Anim. Health Res. Rev. 15:145-147. https://doi.org/10.1017/ S1466252314000267. 
Mellor, D. J., and K. J. Stafford. 2004. Animal welfare implications of neonatal mortality and morbidity in farm animals. Vet. J. 168:118-133. https://doi.org/10.1016/j.tvjl.2003.08.004.

Ollivett, T. L. 2014. Understanding the diagnosis and risk factors for respiratory disease in dairy calves. $\mathrm{PhD}$ Thesis. Department of Population Medicine, University of Guelph, Guelph, Canada.

Ollivett, T. L., and S. Buczinski. 2016. On-farm use of ultrasonography for bovine respiratory disease. Vet. Clin. North Am. Food Anim. Pract. 32:19-35. https://doi.org/10.1016/j.cvfa.2015.09.001.

Ollivett, T. L., J. L. Caswell, D. V. Nydam, T. Duffield, K. E. Leslie, J. Hewson, and D. Kelton. 2015. Thoracic ultrasonography and bronchoalveolar lavage fluid analysis in Holstein calves with subclinical lung lesions. J. Vet. Intern. Med. 29:1728-1734. https://doi .org/10.1111/jvim.13605

Rabeling, B., J. Rehage, D. Döpfer, and H. Scholz. 1998. Ultrasonographic findings in calves with respiratory disease. Vet. Rec. 143:468-471. https://doi.org/10.1136/vr.143.17.468.

Schaffer, A. P., R. L. Larson, N. Cernicchiaro, G. A. Hanzlicek, S. J. Bartle, and D. U. Thomson. 2016. The association between calfhood bovine respiratory disease complex and subsequent departure from the herd, milk production, and reproduction in dairy cattle. J. Am. Vet. Med. Assoc. 248:1157-1164. https://doi.org/10.2460/ javma.248.10.1157.

Sivula, N. J., T. R. Ames, W. E. Marsh, and R. E. Werdin. 1996. Descriptive epidemiology of morbidity and mortality in Minnesota dairy heifer calves. Prev. Vet. Med. 27:155-171. https://doi.org/10 .1016/0167-5877(95)01000-9.
Stanton, A. L., D. F. Kelton, S. J. LeBlanc, J. Wormuth, and K. E. Leslie. 2012. The effect of respiratory disease and a preventive antibiotic treatment on growth, survival, age at first calving, and milk production of dairy heifers. J. Dairy Sci. 95:4950-4960. https: //doi.org/10.3168/jds.2011-5067.

Teixeira, A. G. V., J. A. A. McArt, and R. C. Bicalho. 2017. Efficacy of tildipirosin metaphylaxis for the prevention of respiratory disease, otitis and mortality in pre-weaned Holstein calves. Vet. J. 219:44-48. https://doi.org/10.1016/j.tvjl.2016.12.004.

Tyler, J. W., D. D. Hancock, S. M. Parish, D. E. Rea, T. E. Besser, S. G. Sanders, and L. K. Wilson. 1996. Evaluation of 3 assays for failure of passive transfer in calves. J. Vet. Intern. Med. 10:304-307.

USDA. 2018. Dairy 2014. Health and Management Practices on U.S Dairy Operations. 2014. USDA-APHIS-VS-CEAH-NAHMS, Fort Collins, CO. \#696.0218

\section{ORCIDS}

E. S. Binversie @ https://orcid.org/0000-0002-7091-3599

P. L. Ruegg (1) https://orcid.org/0000-0002-7211-4512

D. K. Combs (ㄴ) https://orcid.org/0000-0001-6628-549X

T. L. Ollivett @ https://orcid.org/0000-0003-2490-5835 